\title{
Etude cinétique des propriétés catalytiques de l'hémocyanine de scorpion
}

\author{
E. QUEINNEC*, M. GARDES-ALBERT**, F. DUCANCEL***,
} M. GOYFFON ${ }^{\star \star \star \star}$, C. FERRADINI ${ }^{\star \star}$ et M. VUILLAUME*

(Manuscrit reçu le 13 novembre 1990)

\begin{abstract}
RÉSUMÉ Les hémocyanines, métalloprotéines à cuivre, ont pour fonction de transporter l'oxygène dissous dans l'hémolymphe de nombreux mollusques et arthropodes. L'oxyhémocyanine de scorpion présente également des propriétés enzymatiques remarquables : peroxydasiques et catalasiques, bien qu'elle ne possède ni fer, ni noyau porphyrinique. L'aspect cinétique de la réaction catalasique est ici décrite pour la première fois. La constante de vitesse de la réaction globale de l'eau oxygénée avec l'hémocyanine a été déterminée, elle est égale à $(2,25 \pm$ 0,25).10 mol-1.l.s $^{-1}$. Par ailleurs, une étude quantitative par radiolyse ipulsée a permis de mettre en évidence la catalyse de la dismutation de l'anion superoxyde par l'hémocyanine à pH 9. La valeur de la constante de vitesse de cette réaction est égale à $3,5.10^{7} \mathrm{~mol}^{-1} .{\mathrm{I} . \mathrm{s}^{-1}}^{-1}$.
\end{abstract}

SUMMARY Hemocyanins are copper proteins which function as oxygen carriers in the haemolymph of molluscs and arthropods. Scorpion's oxyhemocyanin has enzymatic properties : peroxidasic and catalasic activities, although it has neither iron nor porphyrin ring in the active site. The kinetics of the catalasic reaction is described for the first time and the rate constant of the reaction is $(2.25 \pm 0.25) x$ $10^{4} \mathrm{~mol}^{-1} . \mathrm{I.s}^{-1}$. The reaction of superoxide anion with hemocyanin has been studied using pulse radiolysis at $\mathrm{pH}$. The catalytic rate constant is $3.5 . x$ $10^{7} \mathrm{~mol}^{-1}$.I.s ${ }^{-1}$.

\section{INTRODUCTION}

Les hémocyanines sont des métalloprotéines multimériques contenant deux atomes de cuivre par site actif. Sous la forme oxyhémocyanine (fig. 1) elles sont principalement considérées comme transporteurs d'oxygène dans l'hémolymphe d'arthropodes et de mollusques. II est généralement admis que dans le site actif, chaque cation cuivre est relié à trois résidus imidazole d'histidine [13], le quatrième ligand, endogène, est constitué par un phénolate, le cinquième, exogène, provient de l'oxygène

\footnotetext{
* Biochimie et physiologie du développement, CNRS URA 686, Département de biologie, Ecole normale supérieure, 46, rue d'Ulm, 75230 Paris Cedex 05.

** Laboratoire de chimie physique, CNRS UA 400, Université Paris V, 45, rue des SaintsPères, 75270 Paris Cedex 06.

*** Service de biochimie, Département de biologie, Centre d'études nucléaires de Saclay, 91191 Gif-sur-Yvette Cedex.

**** Laboratoire Lerai, Muséum national d'histoire naturelle, 57, rue Cuvier, 75005 Paris.
} 
(sous forme d'un pont peroxyde) $[8,11,19,22]$. II a déjà été montré que cette protéine possède des activités peroxydasiques [17] ainsi qu'une activité catalasique $[12,15,21,30]$. Néanmoins, l'étude cinétique quantitative de la décomposition de l'eau oxygénée par l'oxyhémocyanine n'a jamais été effectuée. Nous nous sommes donc proposés dans un premier temps d'étudier la cinétique de la réaction de l'eau oxygénée avec l'oxyhémocyanine. Dans un deuxième temps, nous avons étudié par radiolyse pulsée l'action spécifique des radicaux $\mathrm{O}_{2}^{-}-$sur cette protéine.

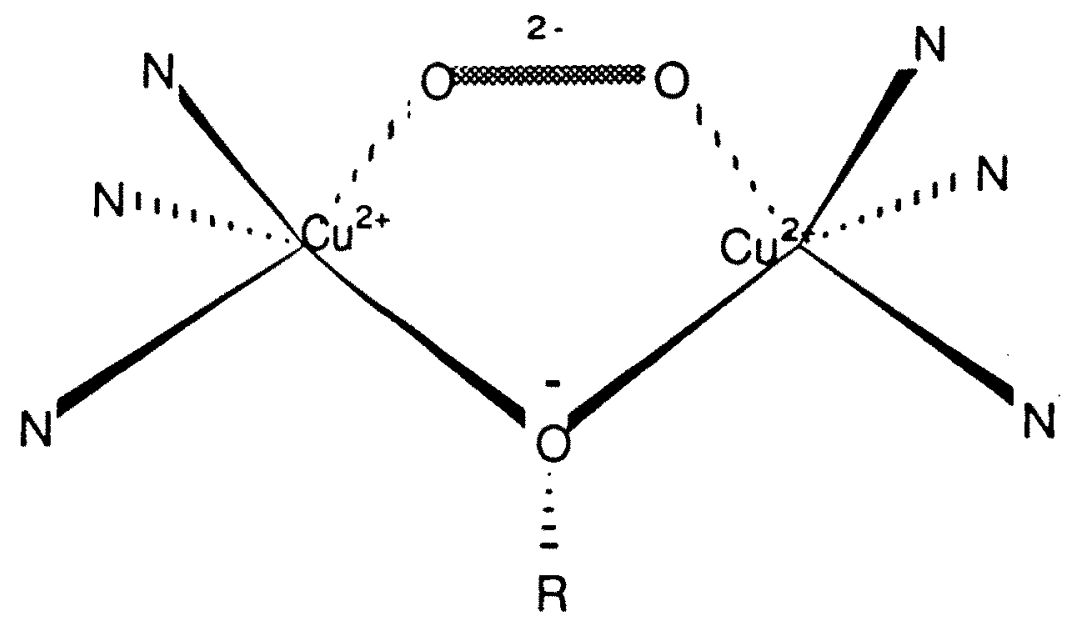

Fig. 1. - Représentation du site actif de l'oxyhémocyanine, d'après T.N. Sorell [18], $N=$ histidine, $\mathrm{H}_{2} \mathrm{O}$ ou liaison vacante, $\mathrm{R}=$ ligand endogène.

\section{MATÉRIEL ET MÉTHODES}

\section{Préparation de l'oxyhémocyanine et de l'apohémocyanine}

L'hémolymphe est prélevée sur le scorpion Androctonus australis par ponction entre deux tergites. La purification de l'hémocyanine s'effectue selon les modalités décrites antérieurement [21, 29]. L'hémocyanine éluée est concentrée par dialyse dans un Micropodicon R120. La concentration en protéine est déterminée par spectrophotométrie d'absorption en utilisant le coefficient spécifique $A_{278} 1 \%=14,76$ [2]. L'apoprotéine est obtenue par dialyse à $+4^{\circ} \mathrm{C}$ contre un tampon : Tris

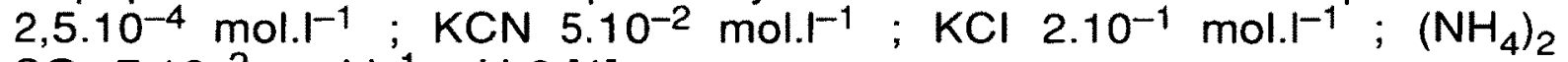
$\mathrm{SO}_{4} 7.10^{-2} \mathrm{~mol}^{-1}, \mathrm{pH} 9[4]$.

\section{Mesure de l'activité catalasique}

L'action de l'eau oxygénée sur l'oxyhémocyanine a été étudiée en mesurant l'oxygène apparu à l'aide d'une électrode de type Clark. La 
concentration d'oxygène saturante dans l'eau de la cuve, sous la pression atmosphérique avant réaction était de $0,265 \mu$ mole. $\mathrm{ml}^{-1}$ à $25^{\circ} \mathrm{C}$. La méthode de calcul de la concentration en oxygène dégagé a été décrite antérieurement [21]. Par ailleurs, l'effet de différents inhibiteurs classiques des catalases : cyanure, aminotriazole (AMT) et azoture de sodium, à une concentration finale de $10^{-3}$ mol..$^{-1}$ a été testé sur l'oxyhémocyanine dont la concentration est de $2.10^{-7} \mathrm{~mol}^{-1} \mathrm{I}^{-1}$.

\section{Mesure de l'activité de type superoxyde dismutase}

Les problèmes posés par la formation de l'anion superoxyde par voie chimique, en particulier une quantification difficile, nous ont conduit à le générer par voie radiolytique.

\section{a) Méthode de la radiolyse et sélection des radicaux superoxyde}

La radiolyse de l'eau entraîne sa décomposition en produits radicalaires $\mathrm{OH}^{*}, \mathrm{e}^{-}$aq, $\mathrm{H}^{*}$ et moléculaires $\mathrm{H}_{2} \mathrm{O}_{2}$ et $\mathrm{H}_{2}$. En présence de fortes concentrations en formiate de sodium $\left(0,16 \mathrm{~mol}^{-1}\right)$ et sous atmosphère d'oxygène, les espèces $\mathrm{OH}^{\cdot}$ et $\mathrm{H}^{*}$ sont converties en radicaux $\mathrm{O}_{2}{ }^{-}$- selon les réactions suivantes:

$$
\begin{array}{lll}
\mathrm{OH}^{\bullet}+\mathrm{HCO}_{2}-\rightarrow \mathrm{H}_{2}+\mathrm{CO}_{2}^{\bullet-} & \mathrm{k}=2,5 \cdot 10^{9} \mathrm{~mol}^{-1} . \mathrm{I} . \mathrm{s}^{-1} \\
\mathrm{H}^{\bullet}+\mathrm{HCO}_{2}-\rightarrow \mathrm{H}_{2}+\mathrm{CO}_{2}^{\bullet-} & \mathrm{k}=5.10^{8} \mathrm{~mol}^{-1} . \mathrm{I} . \mathrm{s}^{-1} \\
\mathrm{e}^{-} \mathrm{aq}+\mathrm{O}_{2} \rightarrow \mathrm{O}_{2}- & \mathrm{k}=1,9.10^{10} \mathrm{~mol}^{-1} . \mathrm{I} . \mathrm{s}^{-1} \\
\mathrm{CO}_{2}^{\bullet-}+\mathrm{O}_{2} \rightarrow \mathrm{CO}_{2}+\mathrm{O}_{2}^{\bullet-} & \mathrm{k}=4,2.10^{9} \mathrm{~mol}^{-1} . \mathrm{I} . \mathrm{s}^{-1}
\end{array}
$$

Ainsi, la radiolyse d'une solution aqueuse oxygénée contenant du formiate de sodium, aboutit à la formation de radicaux $\mathrm{O}_{2}{ }^{-}-$avec un rendement égal à $6,24 \cdot 10^{-7} \mathrm{~mol} \cdot \mathrm{J}^{-1}$.

\section{b) Radiolyse pulsée}

Les irradiations ont été effectuées à l'aide d'un Fébétron 708 (Université Paris V) délivrant des électrons d'énergie de $800 \mathrm{keV}$, la durée de l'impulsion à mi-hauteur étant de 4 ns. Lors de chaque impulsion, les doses délivrées étaient comprises entre $1,0.10^{2}$ et $4,8.10^{2} \mathrm{~Gy}$, ce qui correspond à des concentrations initiales en radicaux $\mathrm{O}_{2}{ }^{-}$comprises entre $6.10^{-5}$ et $3.10^{-4}$ mol. $1^{-1}$. La cinétique de décroissance des radicaux $\mathrm{O}_{2}{ }^{-}$a été suivie par spectrophotométrie d'absorption à $245 \mathrm{~nm}\left(\varepsilon=2300 \mathrm{~mol}^{-1} .1 . \mathrm{cm}^{-1}\right.$ entre $\mathrm{pH} 7$ et 9 , [5], le trajet optique étant de $1 \mathrm{~cm}$. Tous les réactifs utilisés dans ce travail sont garantis " pour analyse ". L'eau était tridistillée (conductivité inférieure à $10^{-6} \Omega^{-1} \cdot \mathrm{cm}^{-1}$ ). 


\section{RÉSULTATS EXPÉRIMENTAUX}

\section{Etude cinétique de la décomposition de l'eau oxygénée par l'oxyhémocyanine}

Sur la figure 2 sont représentées les courbes d'apparition de l'oxygène en fonction du temps observées lors de la réaction de l'oxyhémocyanine avec l'eau oxygénée ([oxyhémocyanine] $=2.10^{-7}$ mol. $\left.\right|^{-1} ;\left[\mathrm{H}_{2} \mathrm{O}_{2}\right]_{0}$ : $8.10^{-4}, 4.10^{-4}, 2.10^{-4}, 8.10^{-5}$ et $8.10^{-6}$ mol. $1^{-1}$; tampon phosphate, $\mathrm{pH} 7,3, \mathrm{t}^{\circ}=25^{\circ} \mathrm{C}$. Le dégagement d'oxygène augmente proportionnellement à la concentration d'eau oxygénée. Aux faibles concentrations en eau oxygénée $\left(\leq 8.10^{-5} \mathrm{~mol}^{-1} \mathrm{I}^{-1}\right.$ ), on note l'existence d'un plateau final (pour $t \approx 180 \mathrm{~s}$ ) correspondant à une concentration finale en oxygène égale à la moitié de la concentration initiale en eau oxygénée :

$$
\left[\mathrm{O}_{2}\right]_{\mathrm{f}}=\left[\mathrm{H}_{2} \mathrm{O}_{2}\right]_{0} / 2
$$

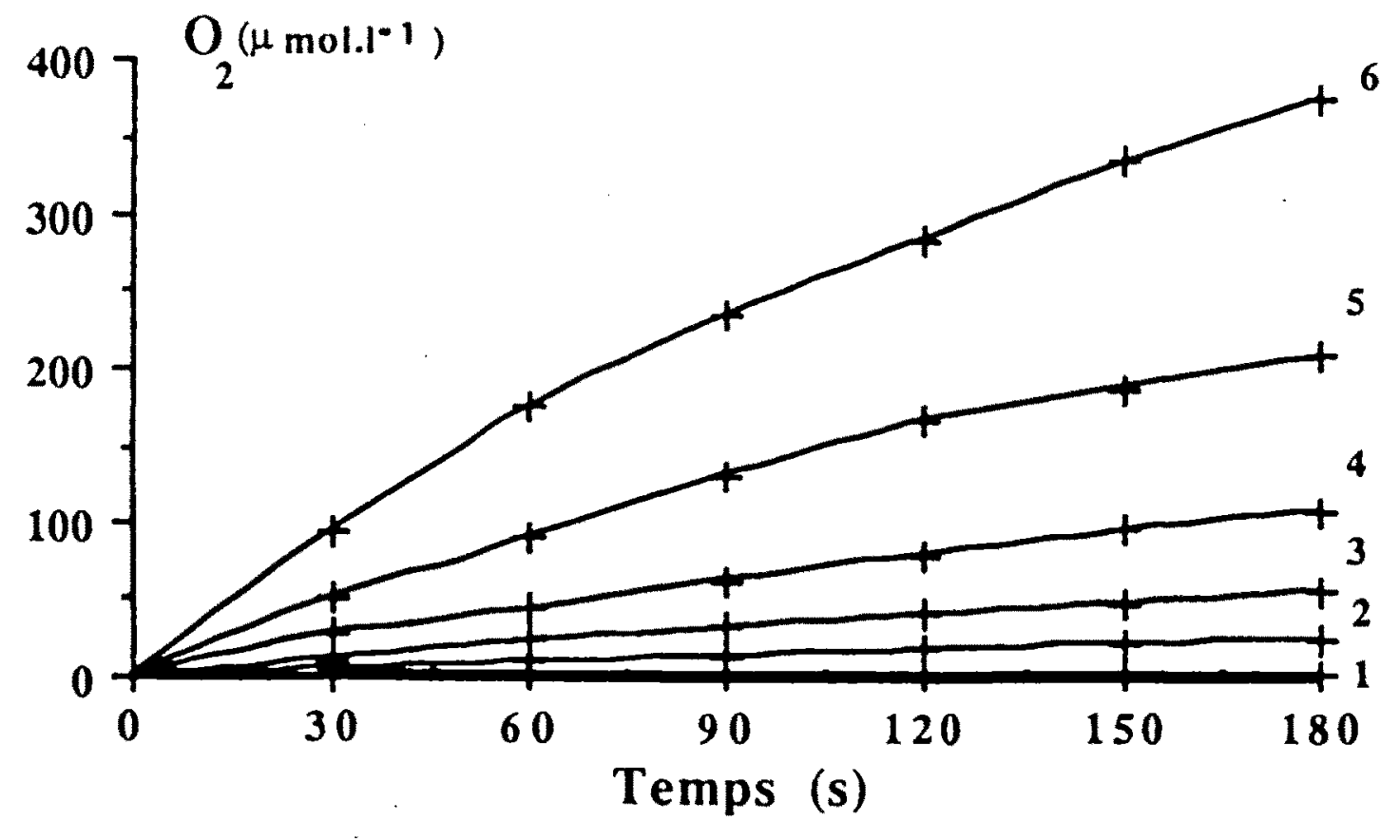

Fig. 2. - Apparition de l'oxygène en fonction du temps au cours de la réaction de l'oxyhémocyanine [(oxyhémocyanine $)_{0}=2.10^{-7}$ mol.t-1] avec l'eau oxygénée. Tampon phosphate, $p H 7,3$. 1) $\left(\mathrm{H}_{2} \mathrm{O}_{2} \mathrm{l}_{0}=8.10^{-6} \mathrm{~mol}^{-1}\right.$; 2) $\left(\mathrm{H}_{2} \mathrm{O}_{2} \mathrm{O}_{\mathrm{O}}=4.1 \mathrm{O}^{-5} \mathrm{~mol} . \mathrm{I}^{-1} ; 3\right)\left(\mathrm{H}_{2} \mathrm{O}_{2}\right)_{0}=8.10^{-5} \mathrm{~mol}^{-1}$; 4) $\left(\mathrm{H}_{2} \mathrm{O}_{2} \mathrm{O}_{0}\right.$ $=2.10^{-4}$ mol.t. $^{-1}$; 5) $\left.\left.\left(\mathrm{H}_{2} \mathrm{O}_{2}\right)_{0}=4.10^{-4} \mathrm{~mol}^{-1} ; 6\right)\left(\mathrm{H}_{2} \mathrm{O}_{2}\right)_{0}=8.10^{-4} \mathrm{~mol}^{-1}\right)$

En supposant que cette relation est vérifiée dans toute la gamme des concentrations en $\mathrm{H}_{2} \mathrm{O}_{2}$ étudiées, la décomposition de l'eau oxygénée correspondrait à la réaction globale de dismutation suivante :

$$
\mathrm{H}_{2} \mathrm{O}_{2}+\mathrm{H}_{2} \mathrm{O}_{2} \rightarrow 2 \mathrm{H}_{2} \mathrm{O}+\mathrm{O}_{2}
$$


De plus, il ne semble pas y avoir de saturation de l'oxyhémocyanine par l'eau oxygénée bien que la concentration en $\mathrm{H}_{2} \mathrm{O}_{2}$ soit élevée $\left.\left(\left[\mathrm{H}_{2} \mathrm{O}_{2}\right]_{0}>\text { [oxyhémocyanine }\right]_{0}\right)$. Les pentes des courbes à l'origine correspondant aux vitesses initiales d'apparition de l'oxygène sont proportionnelles à la concentration en $\mathrm{H}_{2} \mathrm{O}_{2}$ comme on peut le voir sur la figure 3. Par conséquent, la vitesse initiale est donnée par la relation :

$$
\mathrm{Vi}=\left(\mathrm{d}\left[\mathrm{O}_{2}\right] / \mathrm{dt}\right)_{\mathrm{t}=0}=\mathrm{k}_{\mathrm{app}}\left[\mathrm{H}_{2} \mathrm{O}_{2}\right]_{0}
$$

avec $k_{\text {app }}=(4,5 \pm 0,5) \cdot 10^{-3} s^{-1}$.

Une telle réaction est inhibée par le cyanure, mais ne l'est pas par les inhibiteurs classiques des catalases ; aminotriazole, cyanure et azoture de sodium [21]. Par ailleurs, nous avons vérifié que l'apoprotéine ne réagit pas avec l'eau oxygénée.

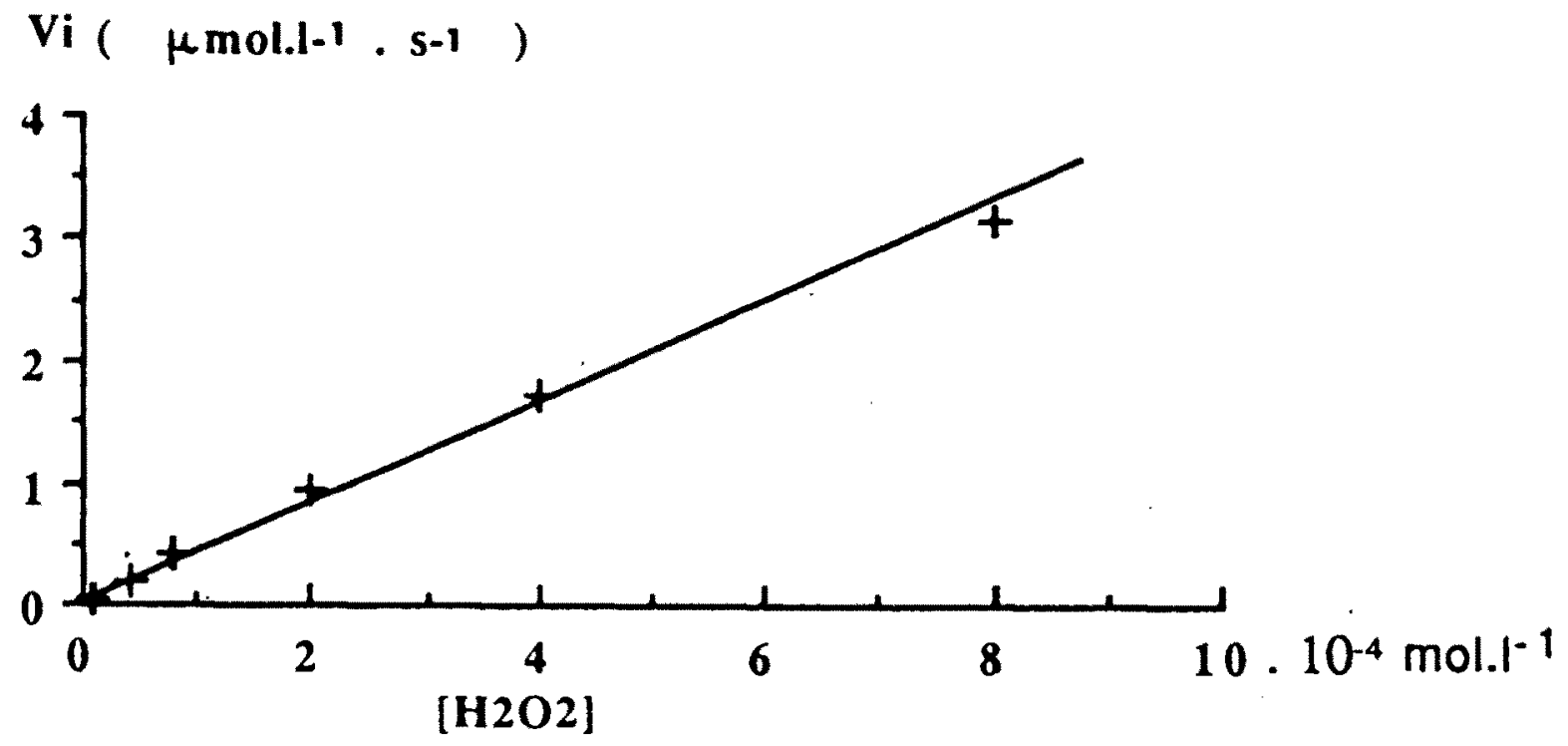

Fig. 3. - Variation de la vitesse initiale d'apparition de loxygène en fonction de la concentration en eau oxygénée. [oxyhémocyanine] $]_{0}=2.10^{-7}$ mol.t ${ }^{-1}$. Tampon phosphate, pH 7,3 .

\section{Etude cinétique de l'action des radicaux superoxyde avec l'oxy- hémocyanine}

Nous avons tout d'abord vérifié qu'en l'absence d'oxyhémocyanine, les radicaux $\mathrm{O}_{2}{ }^{--}$disparaissent selon une loi cinétique du second ordre, avec une constante de vitesse égale à $(1,9 \pm 0,6) \cdot 10^{4} \mathrm{~mol}^{-1} \cdot \mathrm{I}^{-1} \mathrm{~s}^{-1}$ à $\mathrm{pH} 9$ (tampon borate, $5.10^{-4}$ mol. $1^{-1}$, saturé en oxygène). Cette valeur est du même ordre de grandeur que celle que l'on peut calculer d'après la 
constante de vitesse $k=8,5 \cdot 10^{7} \mathrm{~mol}^{-1} .1 . \mathrm{s}^{-1}$ [3] à l'aide de l'expression 2.k.10 $\mathrm{pKa}-\mathrm{pH}$ relative à la réaction bien connue :

$$
\mathrm{O}_{2}^{\cdot-}+\mathrm{HO}_{2} \cdot \stackrel{\mathrm{H}^{+}}{\rightarrow} \mathrm{H}_{2} \mathrm{O}_{2}+\mathrm{O}_{2}
$$

En présence d'oxyhémocyanine $\left(10^{-7}\right.$ ou $2.10^{-7}$ mol. $\left.\mathrm{I}^{-1}\right)$, les radicaux superoxyde disparaissent selon une loi cinétique du premier ordre, comme on peut le voir sur la figure 4. Les pentes des droites ainsi obtenues $\left(k_{\text {app }}\right)$ sont proportionnelles à la concentration d'oxyhémocyanine (encart de la figure 4).

$$
\mathrm{k}_{\mathrm{app}}=\mathrm{k}[\mathrm{oxyHc}]_{0}
$$

On détermine ainsi la valeur de la constante de vitesse globale de réaction de l'oxyhémocyanine avec les radicaux $\mathrm{O}_{2}^{-}-\mathrm{k}_{\text {, égale à }}$ $3,5.10^{7} \mathrm{~mol}^{-1} \cdot \mathrm{I}_{\mathrm{s}}^{-1}$. Une telle valeur a également été mesurée (i) lorsque trois irradiations successives sont effectuées dans une même solution d'oxyhémocyanine ([oxyhémocyanine] $=10^{-7}$ mol..$^{-1} ;$ tampon borate, $5.10^{-4} \mathrm{~mol}^{-1}, \mathrm{pH} 9$, saturé en oxygène, doses par impulsion $\approx 400 \mathrm{~Gy}$ ) et (ii) lorsque le $\mathrm{pH}$ et la concentration en oxyhémocyanine varient $(\mathrm{pH} 8$ 8,5 [oxyhémocyanine] $=10^{-7}, 2.10^{-7}, 5.10^{-7}$ et $10^{-6}$ mol. $1^{-1}[25]$ ).

\section{DISCUSSION}

\section{Décomposition de l'eau oxygénée par l'oxyhémocyanine}

Pour interpréter nos résultats expérimentaux nous proposons le mécanisme simple suivant :

$$
\begin{aligned}
& \mathrm{OxyHC}+\mathrm{H}_{2} \mathrm{O}_{2} \rightarrow \underset{(2 \mathrm{Cu}(\mathrm{I}))}{\mathrm{RedHc}+\mathrm{O}_{2}+\mathrm{H}^{+}} \\
& {[2 \mathrm{Cu}(\mathrm{II})]} \\
& \mathrm{RedHc}+\mathrm{H}_{2} \mathrm{O}_{2} \rightarrow \underset{[2 \mathrm{Cu}(\mathrm{II})]}{\mathrm{OxyHc}+2 \mathrm{OH}^{-}} \\
& (2 \mathrm{Cu}(\mathrm{I}))
\end{aligned}
$$

La réaction (1) est relative à la réduction de l'oxyhémocyanine (2 $\mathrm{Cu}(\mathrm{II}))$ par l'eau oxygénée, qui donne l'hémocyanine réduite $(2 \mathrm{Cu}(\mathrm{I})$ ), par libération d'oxygène. La réaction (2) représente l'oxydation par $\mathrm{H}_{2} \mathrm{O}_{2}$ de cette hémocyanine réduite avec régénération de l'enzyme initiale. La réaction globale catalysée est bien la réaction de dismutation de l'eau oxygénée :

$$
2 \mathrm{H}_{2} \mathrm{O}_{2} \rightarrow \mathrm{O}_{2}+\mathrm{H}_{2} \mathrm{O}
$$

D'après ce mécanisme, la vitesse d'apparition de l'oxygène s'écrit :

$$
\mathrm{d}\left[\mathrm{O}_{2}\right] / \mathrm{dt}=\mathrm{k}_{1}[\mathrm{OxyHc}]\left[\mathrm{H}_{2} \mathrm{O}_{2}\right]
$$


et la vitesse initiale est $\mathrm{Vi}=\left(\mathrm{d}\left[\mathrm{O}_{2}\right] / \mathrm{dt}\right)_{\mathrm{t}=0}=\mathrm{k}_{1}[\mathrm{OxyHc}]_{0}\left[\mathrm{H}_{2} \mathrm{O}_{2}\right]_{0}$ avec $\mathrm{k}_{1}[\mathrm{OxyHc}]_{0}=\mathrm{k}_{\mathrm{app}}$

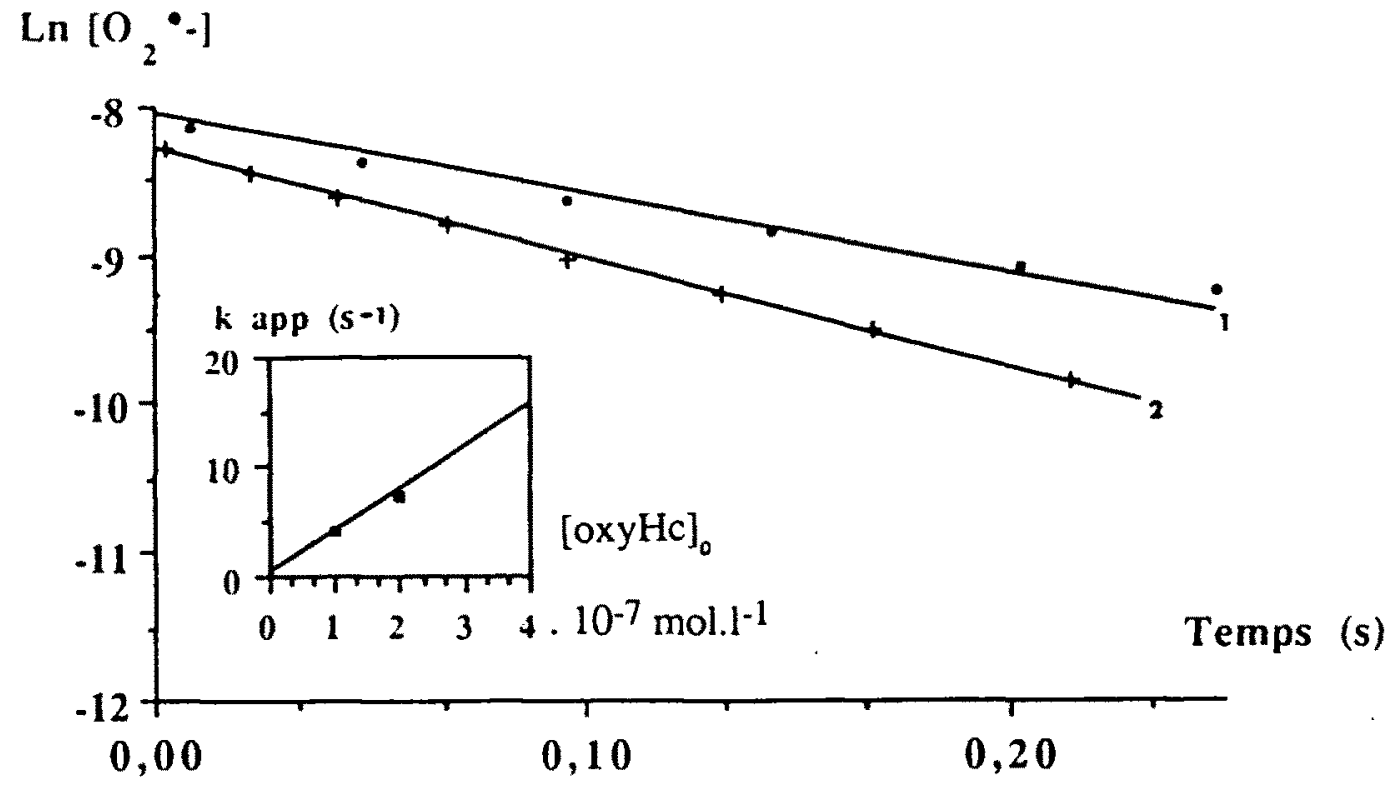

Fig. 4. - Loi cinétique du premier ordre de décroissance de l'anion superoxyde en présence d'oxyhémocyanine. Tampon borate, $5.10^{-4}$ mol.t ${ }^{-1}$, saturé en oxygène.

1) [oxyhémocyanine $]_{Q}=10^{-7}$ mol.t-1 $^{-1}$ dose $464 \mathrm{~Gy},\left[\mathrm{O}_{2}^{--}\right]_{0}=2,9.10^{-4}$ mol.t ${ }^{-1}$; 2) [oxyhémocyanine $]_{0}=2.10^{-7}$ mol. $^{4}$; dose $408 \mathrm{~Gy},\left[\mathrm{O}_{2}^{--}\right]_{0}=2,55.10^{-4} \mathrm{~mol}^{-\mathrm{T}^{-1}}$.

Cartouche : Constante de vitesse apparente du premier ordre en fonction de la concentration en oxyhémocyanine.

$$
\left.\operatorname{Ln}\left(\left(\left[\mathrm{H}_{2} \mathrm{O}_{2}\right]_{0} / 2-\left[\mathrm{O}_{2}\right]\right) \cdot\left(\mathrm{H}_{2} \mathrm{O}_{2}\right]_{0} / 2\right)^{-1}\right)
$$

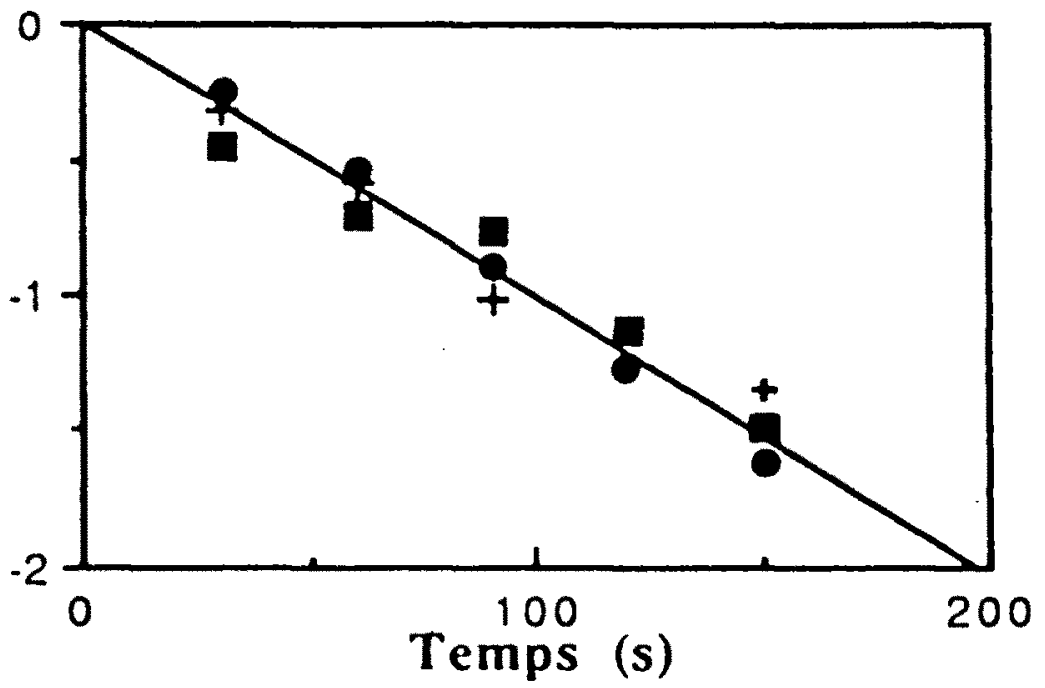

Fig. 5. - Evolution en fonction du temps de l'expression $\left.\mathrm{Ln}\left(\left(\left[\mathrm{H}_{2} \mathrm{O}_{2}\right]_{\sigma^{\prime}}{ }^{2}-\left[\mathrm{O}_{2}\right]_{t}\right) \cdot\left(\mathrm{H}_{2} \mathrm{O}_{2}\right]_{0}{ }^{2}\right)^{-1}\right)$ pour différentes concentrations en eau oxygénée. Tampon phosphate, $\mathrm{pH} 7,3 \cdot[\mathrm{OxyHc}]_{0}=$

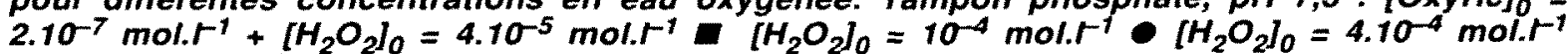


Connaissant la valeur expérimentale de la constante de vitesse apparente, égale à $(4,5 \pm 0,5) \cdot 10^{-3} \cdot \mathrm{s}^{-1}$, on peut en déduire la valeur de $k_{1}=$ $(2,25 \pm 0,25) \cdot 10^{4} \mathrm{~mol}^{-1} \cdot \mathrm{I}_{\mathrm{s}} \mathrm{s}^{-1}$. Une valeur du même ordre de grandeur peut être déterminée à partir de l'expression intégrée donnant l'évolution de $\left[\mathrm{O}_{2}\right]$ en fonction du temps:

$$
\left[\mathrm{O}_{2}\right]_{t}=\left(\left[\mathrm{H}_{2} \mathrm{O}_{2}\right]_{0} / 2\right) \cdot\left(1-e^{-2 k}{ }_{a p p}{ }^{t}\right)
$$

que l'on peut encore écrire :

$$
\left.\operatorname{Ln}\left\{\left(\left[\mathrm{H}_{2} \mathrm{O}_{2}\right]_{0} / 2-\left[\mathrm{O}_{2}\right]_{\mathrm{t}}\right) \cdot\left(\mathrm{H}_{2} \mathrm{O}_{2}\right]_{0} / 2\right)^{-1}\right\}=-2 \mathrm{k}_{\text {app }}{ }^{\mathrm{t}}
$$

Comme on peut le voir sur la figure 5 où sont reportées les valeurs de $\left.\mathrm{Ln}\left(\left(\left[\mathrm{H}_{2} \mathrm{O}_{2}\right]_{0} / 2-\left[\mathrm{O}_{2}\right]_{t}\right) \cdot\left(\mathrm{H}_{2} \mathrm{O}_{2}\right]_{0} / 2\right)^{-1}\right)$ en fonction du temps pour 3 concentrations initiales en peroxyde d'hydrogène, la pente de la droite permet de mesurer une valeur de $k_{\text {app }}$ égale à $(4 \pm 0,5) \cdot 10^{-3} \cdot s^{-1}$, en bon accord avec celle déterminée à partir des vitesses initiales. Remarquons que la constante de vitesse de la réaction de composition de l'eau oxygénée par l'oxyhémocyanine est beaucoup plus faible que celle des catalases classiques $\left(6.10^{6}-2.10^{7} \mathrm{~mol}^{-1} .1 . \mathrm{s}^{-1}[10,14,23]\right)$.

La réactivité de l'hémocyanine vis-à-vis de l'eau oxygénée a déjà fait l'objet de plusieurs travaux [18, 20-21] mais aucun d'entre eux n'a proposé de valeur pour la constante de vitesse de cette catalyse. Toutefois, certains auteurs $[18,20,28]$ ont suggéré un autre schéma réactionnel susceptible d'expliquer qualitativement l'action de l'eau oxygénée sur la déoxyhémocyanine de différents arthropodes. Ce mécanisme est caractérisé par l'existence d'un équilibre (3) entre l'enzyme oxydée (oxyHc) et l'enzyme réduite, déoxygénée (DéoxyHc), indépendemment de la présence de $\mathrm{H}_{2} \mathrm{O}_{2}$ :

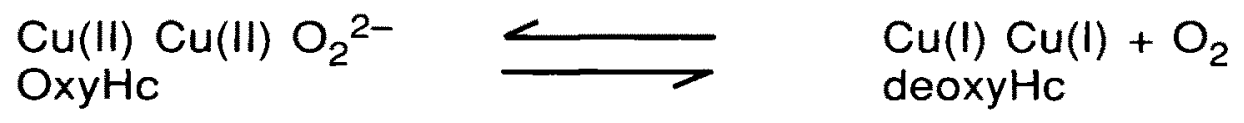

L'action de l'eau oxygénée s'expliquerait alors par le déplacement de cet équilibre vers la droite sous l'effet de la réaction de $\mathrm{H}_{2} \mathrm{O}_{2}$ avec la déoxyhémocyanine. Un tel mécanisme ne peut rendre compte de nos résultats expérimentaux car la vitesse d'apparition de l'oxygène serait d'ordre zéro.

\section{Action des radicaux superoxyde sur l'oxyhémocyanine}

En milieu tamponné à $\mathrm{pH} 9$, les radicaux $\mathrm{O}_{2}^{-}$- réagissent avec l'oxyhémocyanine selon une loi cinétique du premier ordre dont la constante de vitesse est proportionnelle à la concentration d'enzyme. Par ailleurs [25], il a été montré que cette activité ne pouvait être due à du cuivre libre présent en solution ou à un "relargage" après irradiation du cuivre lié de façon spécifique ou non à la protéine. Le mécanisme de cette réaction pourrait être analogue à celui des superoxyde dismutases :

$$
\text { Oxyhémocyanine }+\mathrm{O}_{2}^{\cdot-} \rightarrow \text { Oxyhémocyanine }{ }^{\cdot-}+\mathrm{O}_{2}
$$




$$
\text { Oxyhémocyanine }{ }^{\bullet-}+\mathrm{O}_{2}^{\cdot-} \rightarrow \text { Oxyhémocyanine }{ }^{\cdot-}+\mathrm{H}_{2} \mathrm{O}_{2}
$$

La réaction (4) représente la réduction monoélectronique de l'oxyhémocyanine par les radicaux $\mathrm{O}_{2} \cdot{ }^{-}$. Ces derniers réoxydent la forme transitoire radicalaire $\mathrm{OxyHC}^{\circ}$ - en donnant l'enzyme initiale (réaction (5). L'ensemble de ces deux réactions correspond bien à la dismutation des radicaux $\mathrm{O}_{2}{ }^{--}$. D'après ce schéma réactionnel, l'expression de la vitesse de disparition des radicaux superoxyde s'écrit :

$$
\mathrm{d}\left[\mathrm{O}_{2} \cdot-\right] / \mathrm{dt}=-\mathrm{k}_{\text {cat }}[\mathrm{OxyHc}]_{\text {totale }} \cdot\left[\mathrm{O}_{2} \cdot{ }^{-}\right]
$$

La constante de vitesse $k_{c a t}$ pourrait donc bien être la constante de vitesse du deuxième ordre déduite de nos résultats expérimentaux :

$$
k_{\text {cat }}=3,5 \cdot 10^{7} \mathrm{~mol}^{-1} \cdot \mathrm{I.} \mathrm{s}^{-1}
$$

Notons que si cette valeur est très inférieure à celle des superoxyde dismutases $\left(2,3.10^{9} \mathrm{~mol}^{-1} \cdot 1 . \mathrm{s}^{-1}\right.$ [27]), elle est relativement élevée par rapport à celle obtenue pour de nombreux complexes cuivriques $\left(<10^{6} \mathrm{~mol}^{-1} . \mathrm{I} . \mathrm{s}^{-1}[11-13]\right)$.

\section{CONCLUSION}

L'ensemble des fonctions catalytiques décrites ci-dessus (dismutation de $\mathrm{H}_{2} \mathrm{O}_{2}$ et dismutation de $\mathrm{O}_{2}{ }^{-}$ainsi que la fonction peroxydasique, confèrent à l'hémocyanine de scorpion des propriétés anti-oxydantes particulièrement intéressantes. En effet, bien que les vitesses de disparition de $\mathrm{H}_{2} \mathrm{O}_{2}$ et de $\mathrm{O}_{2}{ }^{--}$catalysées par l'oxyhémocyanine soient de moyenne importance, la forte concentration hémolymphatique du pigment (80-

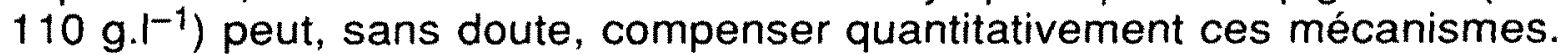
Ces mécanismes pourraient intervenir in vivo pour expliquer la résistance remarquable des scorpions vis-à-vis des rayonnements ionisants.

\section{Remerciements}

Ce travail a bénéficié d'une bourse d'étude de la Société française de radioprotection (EQ) ainsi que d'un contrat EDF-Radioprotection (MV).

\section{RÉFÉRENCES}

[1] ADAMS G.E., WILLSON R.L. - Pulse radiolysis studies on the oxidation of organic radicals in aqueous solution. Trans. Faraday Soc., 1969, 65, 2981-2987.

[2] ANBAR M. - Water and aqueous solutions. In : Fundamental processes in radiation chemistry (P. Ausloos, Ed.). New York : Wiley-Interscience, 1968, 651-679.

[3] BEHAR D., CZAPSKI J., RABANI J., DORFMAN L.M., SCHWARZ H.A. - The acid association dissociation constant and decay kinetics of the perhydroxyl radical. J. Phys. Chem., 1970, 74, 3209-3213. 
[4] BELTRAMINI M., RICHELLI F., SALVATO B. - The kinetics of the reaction of Octopus vulgaris hemocyanin with cianide. Its signifance for the structure of the $11 \mathrm{~S}$ subunit of Molluscan hemocyanin. Inorg. Chem. Acta, 1984, 92, 209-217.

[5] BIELSKI B.H.J., CABELLI D.E., ARUDI R.L. - Reactivy of $\mathrm{HO}^{2 \%} \mathrm{O}_{2}{ }^{- \text {- }}$ radicals in aqueous solution. J. Phys. Chem. Ref. Data, 1985, 14, 1041-1100.

[6] BONNER O.D., WOOLSEY G.B. - The effect of solutes and temperature on the structure of water. J. Phys. Chem., 1968, 72, 899-905.

[7] BRIGELIUS R., SÖTTL R., BORS W., LENGFELDER E., SARAN M., WESER V. - Superoxide dismutase activity of low molecular weight $\mathrm{Cu}^{2+}$ chelates studied by pulse radiolysis. FEBS Lett., 1974, 47, 72-75.

[8] BROWN J.M., POWERS L., KINCAID B., LARRABEE J.A., SPIRO T.G. Structural studies of the hemocyanin active site. 1. Extended $X$-ray absorption fine structure (EXAFS) analysis. J. Am. Chem. Soc., 1980, 102, 4210-4216.

[9] CABELLI D.E., BIELSKI B.H.J., HOLCMAN J. - Interaction between copper(II)arginine complexes and $\mathrm{HO}^{2 \%} / \mathrm{O}_{2} \cdot-$ radicals, a pulse radiolysis study. J. Am. Chem. Soc., 1987, 109, 3665-3669.

[10] CHANCE B. - The enzyme - Substrate compounds of catalase and peroxides. Nature, 1948, 161, 914-917.

[11] EICKMAN N.C., HIMMELWRIGHT R.S., SOLOMON E.I. - Geometric and electronic structure of oxyhemocyanin : spectral and chemical correlations to met apo, half met, met, and dimer active sites. Proc. Natl. Acad. Sci. USA, 1979, 76, 20942098.

[12] FELSENFELD G., PRINTZ M.P. - Specific reactions of hydrogen peroxide with the active site of hemocyanin. The formation of methemocyanin. J. Am. Chem. Soc., $1959,81,6259-6264$.

[13] GAYKEMA W.P.J., HOL W.G.J., VEREIJKEN J.M., SOETER N.M., BAK H.J., BEINTEMA J.J. $-3.2 \AA$ structure of the copper-containing, oxygen-carrying protein Palinurus interruptus haemocyanin. Nature, 1984, 309, 23-29.

[14] GEBICKA L., METODIEWA D., GEBICKI J. - Pulse radiolysis of catalase in solution. Reactions of $\mathrm{O}_{2}{ }^{--}$with catalase and its compound I. Int. J. Radiat. Biol., $1989,55,45-50$.

[15] GHIRETTI F. - The decomposition of hydrogen peroxide by hemocyanin and by its dissociation products. Arch. Biochem. Biophys., 1956, 63, 165-176.

[16] GOLDSTEIN S., CZAPSKI G. - Mechanisms of the dismutation of superoxide catalyzed by the copper(II)phenanthroline complex and of the oxidation of the copper(I)phenanthroline complex by oxygen in aqueous solution. J. Am. Chem. Soc., 1983, 105, 7276-7280.

[17] GOYFFON M. - Effets physiopathologiques de l'irradiation par radiations ionisantes chez le scorpion. Thèse Doct. Sci., Paris VI, 1975.

[18] HEPP A.F., HIMMELWRIGHT R.S., EICKMAN N.C., SOLOMON E.I. - Ligand displacement reactions of oxyhemocyanin : comparison of reactivities of arthropods and molluscs. Biochem. Biophys. Res. Commun., 1979, 89, 1050-1057.

[19] HIMMELWRIGHT R.S., EICKMAN N.C., LUBIEN C.D., SOLOMON E.I. Chemical and spectroscopic comparison of the binuclear copper active site of mollusc and arthropod hemocyanins. J. Am. Chem. Soc., 1980, 102, 5378-5388.

[20] HIMMELWRIGHT R.S., EICKMAN N.C., LUBIEN C.D., LERCH K., SOLOMON E.I. - Chemical and spectroscopic studies of the binuclear copper active site of Neurospora tyrosinase : comparison to hemocyanins. J. Am. Chem. Soc., 1980, 102, 7339-7344.

[21] HUYART N., CALVAYRAC R., BRIAND J., GOYFFON M., VUILLAUME M. Catalatic properties of hemocyanin in helping to account for the scorpion's radioresistance. Comp. Biochem. Physiol., 1983, 76B, 153-159. 
[22] KARLIN K.D., FAROOQ A., HAYES J.C., COHEN B.I., ROWE T.M., SINN E., ZUBIETA J. - Models for Met-hemocyanin derivatives : structural and spectroscopic comparisons of analogous phenolate and $X\left(X=\mathrm{OH}^{-}, \mathrm{OMe}^{-}, \mathrm{N}_{3}^{-}, \mathrm{CL}^{-}, \mathrm{OAC}^{-}\right.$, $\mathrm{OBz}^{-}$) doubly bridged dinuclear copper(II) complexes. Inorg. Chem., 1987, 26, 1271-1280.

[23] KREMER M.L. - israel J. Chem., 1975, 13, 91-98.

[24] OBERLEY L.W., KASEMSET ST-CLAIR D., AUTOR A.P., OBERLEY T.D. Increase in manganese superoxide dismutase activity in the mouse heart after $X$ irradiation. Arch. Biochem. Biophys., 1987, 254, 69-80.

[25] QUEINNEC E., GARDES-ALBERT M., GOYFFON M., FERRADINI C., VUILLAUME M. - Antioxidant activity of hemocyanin ; a pulse radiolysis study. Biochim. Biophys. Acta, 1990, 1041, 153-159.

[26] RABANI J. - On the reactivity of hydrogen atoms in aqueous solutions. J. Phys. Chem., 1962, 66, 361-363.

[27] SORELL T.N. - Synthetic models for binuclear copper proteins. Tetrahedron, $1989,45,3-68$.

[28] TOPHAM R.W., TESH S., BONAVENTURA C., BONAVENTURA J. - Active-site heterogeneity in Limulus hemocyanin as revealed by reaction with peroxides. Arch. Biochem. Biophys., 1988, 261, 299-311.

[29] TOULME J.J., VILLA F., GOYFFON M. - Etude spectroscopique des modifications structurales de l'hémocyanine de scorpion induites par les variations de $\mathrm{pH}$ et l'addition de différents sels. Biochimie, 1976, 58, 681-688.

[30] VUILLAUME M., DUCANCEL F., CALVAYRAC R., RABILLOUD T., HUBERT M. GOYFFON M. - Correlation between the catalase-like activity and $\mathrm{H}^{2} \mathrm{O}^{2}$-ATP production of hemocyanin and its subunits and the high radioresistance of the scorpion Androctonus australis. Comp. Biochem. Physiol., 1988, 92B, 17-23.

[31] WITTERS R., GOYFFON M., LONTIE R. - Etude de l'hémocyanine de scorpion par dichroïsme circulaire. C.R. Acad. Sc., Paris, 1974, 278D, 1277-1280. 